\title{
La creatividad: Un proceso cognitivo, pilar de la educación
}

\author{
Creativity: A cognitive process, pillar of education
}

Criatividade: Um processo cognitivo, pillar da educação

\author{
Méndez Sánchez, María Andrea ${ }^{a}$, Ghitis Jaramillo, Tatiana ${ }^{b}$ \\ ${ }^{a}$ Universidad de La Sabana, Bogotá, Colombia \\ Teléfono: 057-1-8615555. Correo electrónico: mariamesa@unisabana.edu.co \\ bUniversidad de La Sabana, Bogotá, Colombia. \\ Teléfono: 057-1-8615555. Correo electrónico: tatiana.ghitis@unisabana.edu.co
}

\begin{abstract}
RESUMEN
La presente investigación busca caracterizar uno de los procesos cognitivos que dan lugar a la creatividad. Dicho estudio se basó en la propuesta de la escuela de Texas desarrollada por los autores Finke, Ward \& Smith (1996), quienes afirman que los procesos cognitivos creativos se dan en dos fases, la generativa y la exploratoria. El estudio fue cualitativo de tipo descriptivo y buscó caracterizar la transferencia analógica (PCC) en los relatos de ficción escritos por los estudiantes de siete y ocho años de edad participantes del estudio. El análisis permitió demostrar que la educación es el vehículo pertinente y adecuado para el desarrollo de la capacidad creadora en niños y niñas.
\end{abstract}

Palabras clave: educación, creatividad, tarea cognitiva, secuencia didáctica, transferencia analógica.

\begin{abstract}
This investigation is aimed at characterizing one of the cognitive processes that give place to creativity. This study is based on the Texas school proposal, developed by authors Finke, Ward \& Smith (1996), who affirm that cognitive-creative processes are developed in two phases, the generative phase and the exploratory phase. This qualitative and descriptive study is aimed at characterizing the analogical transfer (PCC) in fiction stories written by seven and eight years old students participating in the study. Analysis has allowed the demonstration that education is the pertinent and adequate vehicle for developing the creative capacity in both boys and girls.
\end{abstract}

Key words: education, creativity, cognitive work, didactic sequence, analogical transfer.

\section{RESUMO}

Pretende-se caracterizar um dos processos cognitivos que dão lugar à criatividade. Baseou-se na proposta da escola do Texas desenvolvida por Finke, Ward \& Smith (1996), os quais afirmam que os processos cognitivos criativos se dão em duas fases, a gerativa e a exploratória. Estudo qualitativo do tipo descritivo que procurou caracterizar a transferência analógica (PCC) em histórias imaginárias escritas por estudantes de sete e oito anos participantes do estudo. A análise permitiu demonstrar que a educação é o veículo pertinente e adequado para o desenvolvimento da criatividade em crianças de ambos os sexos.

Palavras chave: educação, criatividade, tarefa cognitiva, sequência didática, transferência analógica.

\section{INTRODUCCIÓN}

La presente investigación pretende la caracterización del proceso cognitivo creativo denominado transferencia analógica, como uno de los pasos que componen la creatividad 
como capacidad cognitiva humana y que entendida como atributo universal a todos les seres humanos, se convierte en un elemento más a tener en cuenta dentro de los procesos de enseñanza en el sistema escolar. Es así que, partiendo de un estudio cualitativo, descriptivo, no experimental, realizado durante el proceso de producción escrita de un relato de ficción de niños y niñas de siete y ocho años de edad se hizo evidente cómo la educación debe buscar la manera de convertirse en el camino para el desarrollo de las capacidades creadoras de los seres humanos.

\section{REFERENTE CONCEPTUAL}

El referente conceptual que sustenta la presente investigación cuenta con tres ejes: la conceptualización de la creatividad, la transferencia analógica como proceso cognitivo creativo y la narración de ficción como elemento didáctico del proceso.

\subsection{CREATIVIDAD}

La conceptualización de la creatividad ha sido abordada por seis modelos explicativos considerados por Stenberg (1999) como, el místico, el psicoanalítico, el pragmático, el psicométrico, el sociopersonal, y el cognitivo; estos enfatizan acerca de aspectos relacionados con las dimensiones personal, sociocultural o cognitiva. Para el presente estudio se tendrá en cuenta el modelo cognitivo, el que se refiere a las representaciones y procesos mentales subyacentes a la creatividad, esta no es resultado de un "único talento o capacidad, sino de la interacción de diversos procesos mentales que todo individuo puede desarrollar y refinar por medio de la experiencia" (Parra, Marulanda, Gómez y Espejo, 2005: 41).

Dentro de este modelo se destacan dos enfoques: el computacional de Margaret Boden (1994) y el de Cognición Creativa o Modelo Geneplore de Finke, Ward \& Smith (1996, 1997). El primero busca brindarle a la creatividad una explicación científica, para lo que se vale de lo afirmado por autores como Poincaré (Cit. en Finke et al., 1997), quien afirmó que la creatividad requiere de la combinación oculta de ideas inconscientes y denominó cuatro fases del proceso creativo, a saber: preparación, intentos conscientes por resolver el problema usando métodos conocidos; incubación, la mente consciente esta concentrada en otras cosas mientras las ideas se combinan con libertad y se dan innovaciones provechosas; iluminación, manifestación del trabajo inconsciente previo; y la verificación, en donde es probada y detallada la manifestación creativa.

De modo similar en la actualidad, la creatividad es entendida como un entrelazamiento de dos habilidades o formas de razonamiento previamente no relacionadas, argumentando que cuanto más inusual es la asociación, más creativa es la idea (Krumm, Vargas-Rubilar y Gullón, 2013). Anterior a esta afirmación, Boden en su modelo computacional argumenta que la creatividad es una capacidad humana tal y como lo es la inteligencia, y que además no es privilegiada para algunos individuos, sino que en general todos los seres humanos la poseen; lo que se requiere es un grado de experticia para lograr potencializarla, ya que se adquiere con persistencia y esfuerzo durante un tiempo considerable y por medio del desarrollo habilidoso y típicamente consciente de un gran número de destrezas psicológicas cotidianas, como observar, recordar y reconocer (Boden, 1994). En este modelo, las ideas deben ser novedosas y valiosas, entendiendo que lo novedoso 
puede ser definido en referencia a ideas previas de la persona o de la totalidad de la historia de la humanidad; y lo valioso hace alusión a que deben ser reconocidas como importantes de alguna manera por el sujeto o desde una negociación por un grupo social a nivel científico, en el ámbito artístico, entre otros.

En cuanto al Modelo Geneplore propuesto por los autores de la Escuela de Texas la creatividad es considerada como un potencial latente que está en dominio de todos los hombres y que puede manifestarse indistintamente en los diferentes campos del conocimiento. Dichos autores afirman que el pensamiento creativo es la actividad humana para la resolución de problemas simples o complejos y, también, es un factor que dinamiza la evolución del hombre (Finke et al., 1997).

Además, los autores de la Escuela de Texas identifican y describen los procesos utilizados por las personas cuya actividad cognitiva es más creativa que la de otras. Es así como proponen diferentes formas de aproximación al estudio de la creatividad teniendo en cuenta diversos puntos de análisis. Un primer acercamiento podría ser mediante la demostración de la existencia de condiciones de novedad así como de propiedades originales, relevantes y pertinentes al problema a solucionar; un segundo acercamiento sería partiendo de la valoración del sujeto que lleva a cabo el acto creativo, bien sea por la presión o restricciones frente a la inhibición de ideas creativas en personas con poca experiencia o la motivación hacia la generación de ideas innovadoras.

Es así como el modelo explica la creatividad haciendo referencia a la articulación de diversos tipos de mecanismos o procesos que mediante dos fases, la generativa y la exploratoria, dan lugar a representaciones mentales internas, estas entendidas como estructuras portadoras de información (estructuras preinventivas), que emergen de la búsqueda creativa y la exploración.

Es importante tener en cuenta que los diferentes procesos y mecanismos que pertenecen a los procesos cognitivos, durante una tarea cognitiva de creación, pueden no presentarse todos, ni en un orden estricto (a excepción del recuerdo que está durante todo el proceso). Las estructuras preinventivas se relacionan con imágenes y contenidos verbales, en este sentido una vez ocurridos los procesos generativos (Gene en geneplore) estas toman diferentes formas, como patrones visuales, mezclas mentales, combinaciones verbales, entre otras (Finke et al., 1997).

El proceso finaliza con la fase exploratoria en la que se realizan las mejoras, que deben ser significativas para darle sentido a las estructuras. Son estos los procesos que interpretan la viabilidad de la idea creada; si la interpretación es positiva, se da paso al producto creativo, de lo contrario, se debe ir a la fase inicial para modificar la estructura existente. Por consiguiente, al entender la creatividad como un proceso cognitivo en el que tienen cabida otros procesos, se hace inevitable el hecho de conocer las características de cada uno de estos procesos que hacen posible la construcción o ejecución de algún acto creativo.

Teniendo en cuenta lo anterior, el presente estudio profundizó en la caracterización de la transferencia analógica, por ser esta un proceso que le permite al individuo tener la posibilidad de llevar la información que ha adquirido previamente a otro contexto que resulta totalmente nuevo para él. Adicionalmente, este es un proceso básico tanto para la solución de problemas como para la generación de nuevas ideas y el aprendizaje; razón que se detectó como necesidad la identificación de sus características en los estudiantes de siete y ocho años de edad y así lograr obtener el 
insumo necesario para la posterior creación de estrategias. Acerca de este proceso se expondrá a continuación.

\subsection{TRANSFERENCIA ANALÓGICA}

La Transferencia Analógica es un proceso cognitivo creativo de carácter generativo, esta es entendida como el traspaso del conocimiento de un dominio ya conocido (dominio fuente) a un dominio nuevo (dominio objetivo), es decir, se refiere a la relación o al conjunto de relaciones de un contexto que es transferido a otro y el resultado se presenta en estructuras preinventivas que son análogas a esas que ya eran familiares (Finke et al., 1997).

Para la comprensión de la Transferencia Analógica como proceso cognitivo creativo, es relevante mencionar que a lo largo de la historia han sido múltiples las definiciones que se han utilizado en el campo educativo, por ejemplo, se le ha denominado como una asociación de ideas, un proceso de inferencia y como una relación de semejanza. No existe una definición única o universal, pero en lo que sí convergen es en la afirmación que este proceso debe considerarse como componente importante del pensamiento, debido a que el razonamiento analógico está relacionado con la inteligencia, el aprendizaje, el proceso de formación de conceptos y la resolución de problemas y es de este último que se desprende la importancia del proceso de transferencia para el proceso cognitivo creativo.

El proceso de transferencia analógica o proceso de transferencia de información relacional de un dominio conocido a otro dominio novedoso tendrá éxito según el grado de semejanza entre ambos (González, Ceacero y Arnau, 1996). El concepto de semejanza entre dos situaciones análogas se entiende como el grado de solapamiento que se puede establecer entre ellas relacionadas con aspectos superficiales o estructurales. Para Holyoak \& Thagard (1997) en la Mente Analógica los elementos estructurales se refieren a la información relativa a los planes y objetivos del problema y los elementos superficiales son el resto de elementos que no están relacionados con la consecución del objetivo del problema.

Así, los efectos que puede ejercer el grado de semejanza sobre los procesos que se dan en la transferencia analógica dan lugar a una dualidad entre lo positivo y lo negativo de la misma transferencia, por esta razón se debe tener en cuenta la edad, la experiencia y el grado de familiaridad de las personas para que se logre realizar una correcta discriminación entre las características superficiales y estructurales. Asimismo, el grado de semejanza estructural o superficial entre dos problemas análogos pertenecientes a dominios semánticamente opuestos interviene en la exactitud y rapidez de la transferencia analógica (González et al., 1996).

Ahora bien, los elementos que componen la transferencia analógica son la recuperación y la extrapolación. En el proceso de recuperación los sujetos recurren a los conocimientos que han adquirido previamente para resolver problemas nuevos, estos conocimientos se activan de manera selectiva para que su recuperación sea eficaz. Por otro lado, el proceso de extrapolación se da al establecer las correspondencias entre los dos problemas y está constituido por dos subprocesos: el primero en donde se da es en el ensamblaje de correspondencias entre elementos de ambos problemas y el segundo en donde se aplican los operadores de solución del dominio fuente para la solución del objetivo (Novick \& Holyoak, 1991). De igual forma, se pueden generar distintos tipos 
de extrapolaciones, desde unas muy rigurosas y con detalles hasta extrapolaciones en las que se establecen pocas relaciones.

Por último, se presenta la conceptualización de narración y ficción, desde los planteamientos del pensamiento narrativo, el texto narrativo y el relato de ficción; considerando que esta fue la base para el diseño de los instrumentos utilizados para la recolección de datos en la investigación.

\subsection{NARRACIÓN Y FICCIÓN}

Desde la perspectiva de la psicología cognitiva se estudian dos modalidades principales del funcionamiento cognitivo que son el pensamiento narrativo y el pensamiento paradigmático o lógico-científico. El primero es el pensamiento de carácter subjetivo e interpretativo en donde se ve la narración como una forma de construcción de relatos a partir del punto de vista del autor; mientras que el segundo es un pensamiento de carácter descriptivo, formal y explicativo. En otros términos, son dos modalidades diferentes de darle sentido a la experiencia y de intentar resolver problemas (Brunner, 1999).

El pensamiento narrativo es el modo más cercano al "sentido común" por el que los seres humanos organizan sus experiencias y construyen significados, gracias a la interacción de unos con otros; es decir, el pensamiento narrativo es aquel que le permite a cada ser humano construir el significado de sus propias experiencias a medida que estas sean narradas unos a otros; en conclusión, esta construcción surge del continuo hecho de actualizar su propia historia (Brunner, 1999). El pensamiento de tipo narrativo se presenta en el desarrollo de manera anterior al pensamiento de tipo conceptual, motivo por el cual "la existencia de una especie de predisposición innata y primitiva en el ser humano hacia la organización y el pensamiento narrativo, que nos permite rápida y fácilmente aprehenderlo y usarlo en la clasificación de la realidad" (Cit. en Santamaría, 2000: 143).

Continuando con lo anterior, la modalidad narrativa del pensamiento es aquella que ordena la experiencia en un espacio y tiempo determinado y utiliza el conocimiento de la realidad diaria y lo transforma, en definitiva, es aquel que se encarga de los deseos, creencias, e intenciones de las personas; siguiendo en esta línea, el pensamiento narrativo es importante, debido a que el pensamiento está basado en la acción e intención humana, así como en la organización de la experiencia; este es el tipo de conocimiento que nos permite construir el mundo y darle sentido a los acontecimientos que en él ocurren.

Al tomar la narración como la forma común de ver los textos, se hace indispensable el estudio de esta desde una perspectiva estructuralista, donde se profundiza en la estructura interna del texto la segmentación, la distribución de la información y las propiedades de la cohesión y coherencia, todo esto desde un enfoque de las teorías textuales de Van Dijk (2005). Así, un texto narrativo puede definirse como un todo finito y estructurado que está compuesto por signos lingüísticos, aquel donde un agente relata una historia; se basa en acontecimientos reales o ficticios, y se hace alusión a elementos como las acciones, los personajes que las llevan a cabo, el tiempo y el espacio donde se desarrollan y cómo se ordenan estos elementos (Contursi y Ferro, 2000).

Una de las formas reconocidas de textos narrativos es el relato, entendido como una secuencia temporal en la que conviven el tiempo de la historia narrada y el tiempo específico del relato. Este se considera como un modo de enunciación que no hace uso de ninguna forma lingüística Contursi y Ferro, 2000). 
El relato además responde a una secuencia narrativa, referida principalmente a "acciones de personas en la que se puede observar la sucesión de eventos, por lo menos un actor sujeto, los predicados que indiquen transformación, un proceso transformacional de unidad de acción compuesto por una situación inicial, un medio y una situación final y la evaluación o moraleja" (Contursi y Ferro, 2000).

Para el abordaje teórico del relato de ficción es primordial definir el término ficción, el que a su vez remite al texto narrativo que posee las características del concepto. En este sentido, se recurre en un primer momento a las definiciones de uso general y común de la palabra, estas se refieren a la acción de fingir y a la clase de obras literarias generalmente narrativas, que tratan de sucesos y personajes imaginarios; en este sentido se puede entender la ficción como la capacidad de representación en la que lo representado solo existe como experiencia imaginaria de seres existentes (Pozuelo, 1993), es decir, que la ficción es la capacidad de simular con el pensamiento la realidad al representar seres y acontecimientos.

Para el presente estudio y con fines específicos de caracterizar la transferencia analógica en relatos de ficción de niños y niñas de 7 a 8 años se entiende ficción como un tipo de representación en la que lo representado solo existe como experiencia imaginaria, es decir, como creación y estructuración de un mundo posible o mundo ficcional, este entendido como un dominio remoto, ajeno y desconocido al universo de la realidad. En la representación que se realiza se genera una realidad autónoma, no necesariamente vinculada al mundo real e incluso contradictoria respecto de sus posibilidades o normas de existencia. Se alude al hecho de poder simular la realidad al presentar seres y acontecimientos que se desarrollan con cierto grado de verosimilitud desde las circunstancias espacio-temporales y el marco de relaciones que libremente diseña el pensamiento del autor.

Otro aspecto fundamental abordado por la literatura moderna para el relato de ficción es llamado Pacto ficcional, este plantea que el receptor de la ficción debe hacer empatía con la narración y aceptar entrar en el mundo imaginario que se le está presentando, sin realizar juicios acerca de la verdad o falsedad de enunciados ficcionales. Es esta aproximación la que permite explicar cómo es posible la realización de afirmaciones ficcionales y cómo se producen los fenómenos en el que los usuarios o receptores interiorizan las reglas de ese mundo posible conservándolas o modificándolas a su vez y crean nuevas ficciones dentro de ese mismo mundo.

Por otra parte, al abordar el tema acerca del relato de ficción y las analogías es necesario mencionar lo planteado por el clásico autor Rodari, quien hace referencia que el arte de inventar historias se fundamenta en una actividad lingüística denominada el binomio fantástico, el que consiste en palabras que se relacionan, y una necesita de la otra para obtener significado, es decir, que la misma obliga a la palabra a "romper" su esquema habitual y a explorar nuevas definiciones (Rodari, 1983). La relación entre estos términos debe hacerse respondiendo a un patrón particular, por ello no puede usarse una palabra al azar, es indispensable que exista una cierta distancia entre ambas, que se distingan entre ellas y que su aproximación suceda de forma moderada. De esta forma, se conlleva a la práctica de la imaginación y se establece una relación, y se conjuga así un elemento fantástico en el que pueden coexistir los dos dispares elementos. La formación de este nexo requiere una labor de extrañamiento, ya que no se toma la conceptualización de la palabra en su uso cotidiano, sino más bien les permite explorar nuevos usos (Rodari, 1983). 


\section{MÉTODO}

El estudio fue realizado bajo los fundamentos de la investigación cualitativa, con un carácter descriptivo y por medio de un diseño no experimental transaccional descriptivo (Hernández, Fernández y Baptista, 2010); por medio de la aplicación de una tarea cognitiva, con el objetivo de caracterizar la transferencia analógica como proceso cognitivo creativo, en la producción de relatos de ficción de estudiantes de siete y ocho años.

El diseño metodológico de la investigación correspondió a un proceso en el que no se manipularon las variables de estudio sino que se observaron en su ambiente natural, es decir, fue de carácter no experimental, en tanto la aplicación de la tarea cognitiva a los estudiantes se llevó a cabo en su situación escolar cotidiana en el aula; y la recolección de la información fue de tipo transaccional puesto que fue obtenida en un momento especifico y único en el tiempo.

Dicha información fue sistematizada, analizada e interpretada cualitativamente a la luz de las conceptualizaciones abordadas en el marco teórico de la presente investigación, especificando las características de la transferencia analógica como proceso cognitivo creativo; dicha descripción permitió la elaboración de las conclusiones finales.

Para la investigación se utilizaron dos instrumentos de recolección de información, los cuales fueron aplicados a los 24 estudiantes muestra del estudio; estos instrumentos fueron diseñados y aplicados, en un primer momento, con el carácter de prueba piloto, que permitió determinar tanto la pertinencia como la validez de las tareas, frente a los objetivos de la presente investigación, y a los que se les realizaron posteriormente las modificaciones necesarias.

A los estudiantes se les aplicaron dos tareas cognitivas en diferentes momentos y en su ambiente natural de trabajo. La primera tarea se denominó Indagación sobre el concepto de ficción, estos tenía como objetivo conocer las concepciones del concepto de ficción que manejaban los estudiantes. La segunda tarea tenía como objetivo la realización de un relato de ficción por parte de cada uno de los estudiantes y constó de dos momentos: una actividad motivacional y la producción escrita del relato de ficción. El primer momento tenía la finalidad de invitar al niño a sumergirse en un contexto de ficción, y crear expectativas frente al segundo momento, que se realizó cuatro días después.

La actividad motivacional fue realizada durante un espacio de clase en el que se incitó a los niños a participar en una "Reunión Intergaláctica de Escritores de Cuentos de Ficción", primero observaron varios dibujos de personajes de ficción, posteriormente debían crear su propio personaje de ficción, el que debían dibujar y describir según las características que le habían asignado. Por último, para el segundo momento, los estudiantes recibieron su personaje de ficción y las instrucciones para la elaboración del escrito en el que su personaje debía ser el protagonista de la historia.

Con el fin de registrar, sistematizar y analizar los datos obtenidos en cada uno de los instrumentos aplicados se diseñaron matrices. En las matrices diseñadas se procedió inicialmente a registrar y analizar los resultados de las tareas individuales, y posteriormente se realizaron generalizaciones e inferencias a partir de las cuales se establecieron las conclusiones del presente estudio.

Para caracterizar el concepto de ficción de los estudiantes se establecieron las siguientes categorías de análisis: 
a. Coherencia del dibujo realizado por el estudiante con el concepto de ficción expresado de manera escrita, teniendo en cuenta que dicha ilustración además de corroborar el contenido del texto, también tuvo como objetivo inicial motivar y ayudar al estudiante a expresar su concepto de ficción.

b. "El concepto de ficción se apoya en"; es decir, que los estudiantes se refieren a la ficción por medio del uso o descripción de elementos específicos como personaje o actante, contexto, acción, otros.

c. Aproximación del estudiante al concepto, alude a los elementos y características que componen la definición dada por el estudiante y la relación de estos frente a la proximidad al concepto considerado en el presente estudio, a partir de las siguientes categorías: "general", si el estudiante describe la ficción a partir del elemento principal de la definición; "específico", el estudiante describe ficción a partir del elemento principal y considera uno o más elementos del concepto de ficción; "otro", cuando el estudiante parte de otros elementos diferentes o adicionales.

Para la descripción del proceso cognitivo creativo de la transferencia analógica se diseñó una segunda matriz, en la que se registraron los resultados obtenidos en la tarea cognitiva correspondiente a la elaboración de relatos de ficción por parte de los estudiantes y los dos momentos que la conformaron. Las categorías de análisis de la primera actividad son:

Tabla 1.

\begin{tabular}{|l|l|l|}
\hline Categoría & \multicolumn{1}{|c|}{ Indicadores } & \multicolumn{1}{c|}{ Descripción } \\
\hline \multirow{2}{*}{ Proceso de extrapolación } & $\begin{array}{l}\text { Existencia de la } \\
\text { correspondencia }\end{array}$ & $\begin{array}{l}\text { La tarea evidencia correspondencia entre el do- } \\
\text { minio fuente y el dominio objetivo }\end{array}$ \\
\cline { 2 - 3 } & $\begin{array}{l}\text { Inexistencia de la } \\
\text { correspondencia }\end{array}$ & En la tarea no se evidencia la correspondencia \\
\hline \multirow{2}{*}{$\begin{array}{l}\text { Semejanza o grado de so- } \\
\text { lapamiento entre dominio } \\
\text { fuente y dominio objetivo }\end{array}$} & $\begin{array}{l}\text { Semejanza } \\
\text { estructural }\end{array}$ & $\begin{array}{l}\text { En el personaje de ficción realizado se evidencia } \\
\text { el uso de información relativa a planes y objetivos } \\
\text { de la tarea planteada }\end{array}$ \\
\cline { 2 - 3 } & superficial & $\begin{array}{l}\text { En el personaje de ficción se evidencia el uso de } \\
\text { información de otros elementos no relacionados a } \\
\text { la consecución del objetivo de la tarea cognitiva } \\
\text { planteada, es poco riguroso y detallado }\end{array}$ \\
\hline
\end{tabular}

Por último, las categorías de análisis planteadas para los relatos de ficción son: 
Tabla 2.

\begin{tabular}{|c|c|c|c|}
\hline Categoría & \multicolumn{2}{|c|}{ Indicadores } & Descripción \\
\hline \multirow{4}{*}{ Proceso de extrapolación } & \multicolumn{2}{|c|}{$\begin{array}{l}\text { Existencia de la } \\
\text { correspondencia }\end{array}$} & $\begin{array}{l}\text { Cuando en el relato se evidencian correspondencias } \\
\text { entre el dominio fuente y el dominio objetivo, } \\
\text { estableciendo la frecuencia de aparición de este } \\
\text { proceso en los siguientes rangos } 1-2,3-4,5-6,7-8\end{array}$ \\
\hline & \multirow{3}{*}{ Novedad } & Alta & $\begin{array}{l}\text { "Alta", en el relato se evidencia novedad u ori- } \\
\text { ginalidad en el dominio objetivo respecto del } \\
\text { dominio fuente }\end{array}$ \\
\hline & & Media & $\begin{array}{l}\text { "Media", en el relato se evidencia un intento de } \\
\text { redefinición u originalidad del dominio objetivo } \\
\text { respecto del dominio fuente }\end{array}$ \\
\hline & & Baja & $\begin{array}{l}\text { "Baja", en el relato del estudiante no se evidencia } \\
\text { originalidad o novedad en el dominio objetivo } \\
\text { respecto del dominio fuente }\end{array}$ \\
\hline \multirow[t]{2}{*}{$\begin{array}{l}\text { Semejanza o grado de } \\
\text { solapamiento }\end{array}$} & \multicolumn{2}{|c|}{$\begin{array}{l}\text { Semejanza } \\
\text { estructural }\end{array}$} & $\begin{array}{l}\text { En la escritura del relato se evidencia el uso de } \\
\text { información relativa a planes y objetivos de la tarea } \\
\text { cognitiva planteada, es decir, la extrapolación es de } \\
\text { carácter riguroso y hace uso de detalles }\end{array}$ \\
\hline & \multicolumn{2}{|c|}{$\begin{array}{l}\text { Semejanza } \\
\text { superficial }\end{array}$} & $\begin{array}{l}\text { En la escritura del relato se evidencia el uso de } \\
\text { información de otros elementos no relacionados con } \\
\text { la consecución del objetivo de la tarea planteada, es } \\
\text { decir, la extrapolación hace uso de pocos detalles } \\
\text { y no es rigurosa. }\end{array}$ \\
\hline
\end{tabular}

Nota Aclaratoria categoría Proceso de extrapolación: en el análisis se explicitan los dominios de las transferencias utilizadas en los relatos; en este punto es importante tener en cuenta que los dominios se infirieron en el relato, teniendo en cuenta conocimientos previos de los estudiantes, como los relativos a las unidades de indagación del grado $2^{\circ}$, así como su experiencia cotidiana (televisión, películas, libros, entre otros).

\section{RESULTADOS Y DISCUSIÓN}

Una vez realizado el análisis del presente estudio, con los veinticuatro (24) estudiantes de siete y ocho años participantes, se evidenció en 21 de ellos el uso de transferencias analógicas como proceso cognitivo creativo en la producción de relatos de ficción. En este sentido y de acuerdo con el objetivo general de esta investigación, se puede concluir que las transferencias analógicas se caracterizan por ser un proceso que tiene sus inicios en las primeras etapas de la vida y por eso se convierte en un proceso de pensamiento que necesita ser estimulado y trabajado desde los primeros niveles de la educación.

A continuación los resultados del análisis del estudio: 
Figura 1. Proceso de extrapolación

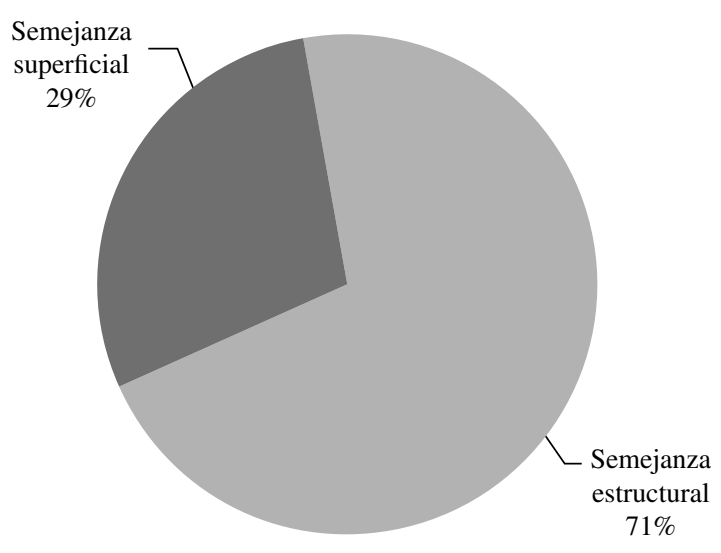

El $71 \%$ de los estudiantes extrapolan generando semejanzas estructurales, ya que al realizar sus relatos de ficción evidencian el uso de información detallada y rigurosa, generando así una solución novedosa a la tarea cognitiva planteada (ejemplo: el conocimiento de una jungla y construir un hogar al interior de un planeta con características de jungla). El resto de la población (29\% de los estudiantes) en sus relatos evidencian que las semejanzas en las extrapolaciones son de tipo superficial, debido a que solo hacen uso de pequeños detalles o características de aquello que conocen con miras a generar una situación o cosa nueva para dar solución a la tarea cognitiva.

En cuanto a la novedad, la mayor parte de los estudiantes que realizaron extrapolaciones de tipo estructural se ubicaron en un indicador medio (10 de 15 estudiantes), porque se observó un intento de redefinición u originalidad del dominio objetivo respecto del dominio fuente; por otro lado solo 5 estudiantes se ubicaron en un indicador alto que implica novedad en el dominio fuente y 6 estudiantes en un indicador bajo. Lo anterior es coherente con lo referido en Aprendizaje por analogía (González et al., 1996), respecto de tener en cuenta la edad, experiencia y el grado de familiaridad de las personas frente a la exactitud de la transferencia analógica y la correcta discriminación de las semejanzas estructurales y superficiales.

Las transferencias analógicas observadas en los relatos de ficción parten de dominios fuentes correspondientes a los conocimientos previos de los estudiantes, como la información y conceptos estudiados en las diferentes unidades de indagación de su programa académico, como por ejemplo: sistema solar, cambios climáticos, atributos de un buen ciudadano, diferentes estilos de vida alrededor del mundo, historia familiar, entre otros; igual que de experiencias cotidianas, programas de televisión, películas y libros. Se observó que la mayoría de los estudiantes aludieron a personajes como superhéroes, contextos interplanetarios y acciones ficcionales al momento de realizar su relato.

La transferencia analógica por sus connotaciones puede llegar a considerarse como un proceso de mayor complejidad; sin embargo, los niños y niñas evidenciaron el uso 
de este proceso en la realización de sus dibujos y relatos, esto debido a su conocimiento de la temática (ficción).

En el proceso de extrapolación se generan con mayor facilidad correspondencias cuando las relaciones son de tipo pictórico; es decir, se ven más semejanzas de tipo estructural en la realización de los dibujos de los personajes de ficción que al momento de escribir los relatos; conclusión correspondiente al análisis adicional realizado a la primera parte de la tarea cognitiva.

Referente a los instrumentos utilizados para la recolección de datos de esta investigación, además del relato de ficción cuyas conclusiones de resultados se expresaron anteriormente, vale la pena mencionar que tanto la tarea de indagación del concepto de ficción como la de motivación en la tarea principal, es decir, la creación del personaje de ficción, arrojaron datos importantes que permitieron establecer otras conclusiones valiosas, como que la tarea de indagación del concepto de ficción manejado por los estudiantes permitió observar cómo dicho concepto fue utilizado como dominio fuente de sus relatos, en tanto se evidenciaron extrapolaciones que partían de dicho conocimiento.

Además se puede afirmar la existencia de una correspondencia entre los estudiantes que tenían un concepto más específico de ficción y aquellos que realizaron transferencias de tipo estructural; en la tarea de creación del personaje de ficción la mayoría de los estudiantes (23) realizaron procesos de extrapolación, de ellos el 75\% fueron semejanzas de carácter estructural; evidenciándose así una correspondencia entre este número de estudiantes y los que en su relato de ficción mostraron también la realización de transferencias analógicas.

Teniendo en cuenta la metodología escogida para el desarrollo del estudio se pudo concluir que fue la adecuada, por ser un estudio en el que se pretendía no sacar a la población de su contexto natural, sino generar un conocimiento de utilidad para la mejora de las actividades en el aula con miras al desarrollo de la creatividad como capacidad humana. Además, el uso de la secuencia en las actividades y los instrumentos utilizados permitió al grupo investigador encontrar el camino para la caracterización del proceso y de una estrategia didáctica para el desarrollo de la creatividad.

Además, durante el análisis se hizo necesaria la caracterización de la ficción de los estudiantes, ya que este fue uno de los dominios objetivos evidenciados, es decir, que todos realizaron sus extrapolaciones partiendo del conocimiento que tenían para construir una historia de ficción propia. Fue así como se encontraron las siguientes características:

- Veintiún estudiantes del presente estudio conocen con claridad el concepto de ficción, aunque no se refieran a este en los términos (técnicos) contemplados en la investigación. Es decir, no se refleja una clara definición debido a que se expresan mediante el uso de ejemplos y de analogías.

- Entre más específico es el concepto de ficción escrito por los estudiantes mayor número de transferencias realizan al interior de su relato, es decir, entre más claridad del dominio objetivo, mayor posibilidad de construir un relato novedoso a partir de aquello que ellos conocen sin salirse de su objetivo: construir una historia ficcional.

- Dos estudiantes hicieron alusión al pacto ficcional, evidenciando un mayor conocimiento del concepto de ficción.

- Dieciocho estudiantes aludieron a personajes para dar su definición, es decir, debido a su nivel de concreción hacen uso de la ejemplificación, por medio de aquellos 
personajes que ellos ya conocen y asocian como ficcionales, ya que no pueden llegar a una definición como tal.

- Once estudiantes hicieron referencia al contexto para dar su definición, un gran porcentaje asoció la ficción con aquellos lugares donde ellos han visto historias de ficción como son las películas, video, juegos, etc. Haciendo evidente su nivel de concreción a la hora de comprender los conceptos.

- Dieciocho estudiantes acudieron a acciones para dar su definición, es decir, un alto porcentaje de los participantes de estudio asocian la ficción a hechos o situaciones, que se saldrían de lo que es el mundo tangencial o real.

- Todos los estudiantes hacen alusión a representaciones de un mundo ficcional, la mayoría ejemplifica con aquellos personajes que ellos conocen.

Por último es importante mencionar que los estudiantes participantes en la presente investigación evidenciaron que al escribir un relato acerca de un tema de su interés, su rendimiento fue mejor que en otras actividades de la normalidad de sus clases, esto fue corroborado mediante lo expresado por la docente del salón, quien afirmó al finalizar la actividad: "muchos de los niños escribieron cuentos mucho más largos y con mejor estructura de lo que generalmente hacen en mi clase". Es decir, se puede afirmar que aunque las secuencias didácticas ya existan, lo que es valioso rescatar de las actividades propuestas para el presente estudio es el darse cuenta que este tipo de práctica pedagógica es válida para el trabajo de la creatividad al interior de las actividades del aula, incluso del currículo de la institución educativa.

\section{CONCLUSIONES}

Una vez finalizado el estudio, el grupo investigador observó la necesidad de continuar con la elaboración de este tipo de estudios haciendo referencia a otros procesos cognitivos creativos con el mismo tipo de tareas cognitivas, con miras a la mejora de los procesos de enseñanza por parte de los docentes para el desarrollo de procesos de pensamiento de sus estudiantes.

De igual forma, se hizo evidente la importancia de realizar en el futuro estudios, que en continuidad al presente, posibiliten la comparación del uso de la Transferencia Analógica como proceso cognitivo, tanto en un relato escrito como en uno oral, con miras a buscar aquella estrategia donde se da de manera más adecuada o con mayor frecuencia dicho proceso, teniendo presente las características propias del desarrollo cognitivo de la edad de los estudiantes.

El grupo investigador reconoce que el uso y la aplicación de las tareas diseñadas para este estudio, que no se alejan de la realidad cotidiana de un aula de clase, permiten desarrollar y fortalecer la creatividad como proceso cognitivo válido y relevante a considerar como eje de trabajo al interior de cualquier currículo escolar. Adicionalmente, es importante resaltar que este tipo de actividades, en tanto secuencia didáctica de trabajo, permite que los docentes se acerquen a aquello que el aprendizaje significativo define como intereses.

La aplicación de la tarea cognitiva diseñada para el estudio, cuyas finalidades eran evidenciar didácticamente el uso de las transferencias analógicas como proceso cognitivo 
creativo en estudiantes de siete a ocho años y describir las características de esta, evidenció durante el análisis de resultados la importancia de reflexionar respecto de la necesidad de buscar que las actividades en el aula le permitan al estudiante hacer uso de sus intereses, con miras a lograr los objetivos académicos propuestos; y el trabajo en creatividad, le permite al docente acercarse a esto.

Otro aspecto relevante a considerar es la importancia de realizar procesos de capacitación a docentes respecto a los procesos cognitivos creativos y posibles estrategias pedagógicas, como la secuencia didáctica propuesta, debido a su importancia para el adecuado desarrollo integral de los estudiantes.

Por último, es valioso rescatar la necesidad de realizar estudios similares acerca de otros procesos cognitivos creativos, buscando en construir un conocimiento que permita hacer aportes valiosos en los procesos de pensamiento de los estudiantes y de esta manera poder mejorar las prácticas de aula en pro del adecuado desarrollo de los mismos.

\section{REFERENCIAS BIBLIOGRÁFICAS}

Boden, M. (1994). La mente creativa: Mitos y mecanismos. Barcelona: Gedisa.

Bruner, J. (1999). Realidad mental y mundos posibles: Los actos de la imaginación que dan sentido a la experiencia. Barcelona: Gedisa.

Contursi, M. y Ferro, F. (2000). La narración. Usos y teorías. Bogotá: Norma.

Finke, R., Ward, T. \& Smith, S. (1997). The creative cognition approach. Cambridge: MIT Press. (1996). Creative cognition. Theory, research, and applications. Cambridge: The MIT Press.

González, L., Ceacero, C. y Arnau, B. (1996). Aprendizaje por analogía: Análisis del proceso de inferencia analógica para la adquisición de nuevos conocimientos. Madrid: C.I.D.E.

Hernández, R., Fernández, C. y Baptista, P. (2010). Metodología de la investigación. Bogotá: McGraw Hill.

Holyoak, K. J. \& Thagard, P. (1997). The analogical mind. American Psychologist, vol. 52 (1), 35-44.

Krumm, G., Vargas-Rubilar, J. y Gullón, S. (2013). Estilos parentales y creatividad en niños escolarizados. Psicoperspectivas. Individuo y Sociedad, vol. 12 (1), 161-182.

Novick, L. R. \& Holyoak, K. J. (1991). Mathematical problem solving by analogy. Journal of Experimental Psychology: Learning, Memory, and Cognition, vol. 17 (3), 398-415.

Parra, J., Marulanda, E., Gómez, F. y Espejo, V. (2005). Tendencias de estudio en cognición, creatividad y aprendizaje ( $1^{\text {ra }}$ Ed.). Bogotá: Javegraf.

Pozuelo, J. (1993). Poética de la ficción. Madrid: Síntesis.

Rodari, G. (1983). Gramática de la fantasía. Introducción al arte de inventar historias. Barcelona: Editorial Argos Vergara.

Santamaría, A. (2000). La narración como acción mediada en el marco de una psicología cultural. Un estudio en adultos. Anuario de Psicología, vol. 31 (4), 139-161.

Stenberg, R. (Ed.). (1999). Handbook of creativity. Cambridge: Cambridge University Press.

Van Dijk, T. (2005). Las estructuras y funciones del discurso: Una introducción interdisciplinaria a la lingüística del texto y a los estudios del discurso (14 ${ }^{\text {ta }}$ Ed.). México D.F: Siglo XXI. 
